\title{
Effect of Biopolymers on Structure and Ice Recrystallization in Dynamically Frozen Ice Cream Model Systems
}

\author{
A. Regand and H. D. Goff \\ Department of Food Science, \\ University of Guelph, Guelph, \\ ON, N1G 2W1, Canada
}

\section{ABSTRACT}

Ice crystal growth and microstructure of sugar solutions prepared with stabilizers (carboxymethyl cellulose [CMC], xanthan gum, locust bean gum [LBG], and gelatin) with or without milk solids-nonfat (MSNF) after freezing in a scraped surface heat exchanger and temperature cycling ( 5 cycles from $-6^{\circ} \mathrm{C}$ to $-20^{\circ} \mathrm{C}$ ) were studied. Ice crystal growth was calculated from brightfield microscopic images acquired from samples before and after cycling. Freeze-substitution and low-temperature embedding (LR-Gold resin) were sample preparation techniques utilized for structure analyses by light microscopy and transmission electron microscopy. Differential staining for carbohydrates and proteins allowed the identification of stabilizer gel-like structures in LBG, gelatin, and gelatin/MSNF solutions. In the absence of milk proteins, xanthan and LBG were the most effective at retarding recrystallization, while in their presence, only xanthan had an effect. Cryo-gelation of the LBG was observed but is not the only mechanism of stabilizer action. Thermodynamic incompatibility between biopolymers was observed to promote localized high concentrations of milk proteins located at the ice crystal interface, probably exerting a water-holding action that significantly enhanced the stabilizer effect. Qualitatively, solution heterogeneity (phase separation) was directly proportional to ice crystal growth inhibition. It is suggested that water-holding by stabilizer and proteins, and in some cases steric hindrance induced by a stabilizer gel-like network, caused a reduction in the kinetics of the ice recrystallization phenomena and promoted mechanisms of melt-regrow instead of melt-diffuse-grow recrystallization, thus resulting in the preservation of the ice crystal size and in a small span of the ice crystal size distribution.

(Key words: ice recrystallization, stabilizer, microscopy, and ice cream).

Received February 16, 2002.

Accepted April 9, 2002

Corresponding author: H. D. Goff; e-mail: dgoff@uoguelph.ca.
Abbreviation key: CMC = carboxymethyl cellulose, LBG = locust bean gum, $\mathbf{L M}=$ light microscopy, $\mathbf{M S N F}$ $=$ milk solids-nonfat, $\mathbf{T E M}=$ transmission electron microscopy.

\section{INTRODUCTION}

Hydrocolloid stabilizers are widely used in ice cream because they hinder ice crystal growth as temperature fluctuates during storage. The mechanisms by which they affect the freezing properties or limit recrystallization have been extensively studied but are still not fully understood. Stabilizers have little (Caldwell et al., 1992a,b) or no (Sutton and Wilcox, 1998a,b) impact on the initial ice crystal size distribution in ice cream at the time of draw from the scraped surface heat exchanger, nor on the initial ice growth during quiescent freezing and hardening (Flores and Goff, 1999a). However, they do limit the rate of growth of ice crystals during recrystallization (Donhowe and Hartel, 1996a,b; Sutton and Wilcox, 1998a,b; Flores and Goff, 1999a,b).

Many studies have been conducted to correlate enhanced viscosity caused by stabilizers with better control of ice crystal growth (Budiaman and Fennema, 1987; Hagiwara and Hartel, 1996; Miller-Livney and Hartel, 1997) but without definitive conclusions. The functionality of a given stabilizer may be enhanced as the polymer concentration is increased, but different stabilizers are not equally effective for retarding ice crystal growth at the same level of viscosity. A different approach taken by Bolliger et al. (2000) found a linear relationship between a normalized "breakpoint" apparent viscosity (the viscosity at which a significant change in slope of concentration-viscosity occurred) and recrystallization rate. They suggested that some aspects of stabilizer functionality could come from the rheology that results from freeze-concentration of the polysaccharide in the unfrozen phase of ice cream. This concept was related to the rate at which water can diffuse to the surface of a growing crystal during temperature fluctuation or the rate at which solutes and macromolecules can diffuse away from the surface of a growing ice crystal (Caldwell et al., 1992a, 1992b; Goff et al., 
1993). Experiments done with nuclear magnetic resonance showed that an increase in viscosity due to the stabilizer was not accompanied by a similar decrease in the mobility of small molecules (i.e., water or sugar [Martin et al., 1999]). However, it is important to remember that by this technique the water diffusion or translational displacement of water (or sugar) molecules is being measured at intermolecular distances, usually less than $10 \mathrm{~nm}$, while the water migration from one crystal to another involved in melt-regrow recrystallization mechanisms implies distances between ice crystals usually longer than $10 \mu \mathrm{m}$. Therefore, the question formulated by Miller-Livney and Hartel (1997) still remains as to whether stabilizers have different effects on macroviscosity (bulk fluid mobility), compared with microviscosity (mobility of constituents in the space surrounding the ice crystal), and whether increased microviscosity retards diffusion of water and sugar molecules.

Recrystallization has been also related to the capacity of the stabilizer to form cryogels or entangled networks during freezing-thawing cycles. Gel firmness has been associated with inhibition of ice crystal growth and a change in ice crystal morphology (Blond, 1988; Muhr and Blanshard, 1986). However, a firm gel has not always been effective at retarding ice crystal growth, probably because a firm gel would be more fragile and more easily ruptured by the ice front, whereas a more flexible gel would exert a stronger opposing force for ice front propagation. It has also been reported that stabilizers that do not form a gel still have an effect in retarding ice crystal growth (Sutton and Wilcox, 1998a). Goff et al. (1999) modified a method to bond covalently a fluorescent marker to locust bean gum (LBG) and guar gum in order to visualize the location of these polysaccharides in frozen sucrose or sucrose/skim milk powder solutions after temperature cycling. They found that the formation of an LBG network, combined with the presence of phase-separated protein, was effective at controlling ice recrystallization. Based on these observations, it was suggested that the ability to form cryo-gels, or gel-like intermolecular interactions, and to interact with proteins, perhaps causing localized increases in concentration through phase-separation, are prerequisites for stabilizer effectiveness against ice recrystallization.

As an extension of the previous work of Goff et al. (1999), other commonly used stabilizers-carrageenan, sodium alginate, carboxymethyl cellulose (CMC), xanthan gum and gelatin, plus LBG, previously studiedwere recently analyzed (Regand and Goff, accepted) to verify a correlation between their capacity to form cryogels and their ability to retard recrystallization when used as stabilizers in ice cream model solutions quies- cently frozen (i.e., using a cold stage). It was found that some nongelling stabilizers were more effective retarding recrystallization than gelling stabilizers, suggesting that steric blocking of the interface or inhibition of solute transport to and from the ice interface caused by gelation of the polymer is not the only mechanism of stabilizer action. However, molecular interactions between polysaccharides and proteins appeared to be key factors in retarding ice recrystallization. It was suggested that water-holding by stabilizers and proteins, and/or steric hindrance by network structures, reduced the kinetics of diffusion in the system, promoting ice recrystallization mechanisms of melt-regrow (hence maintenance of unfrozen water during warming in close proximity to the crystal, so that the water refreezes onto the same crystal during cooling) instead of melt-diffuse-grow (unfrozen water migrates to larger crystals where it refreezes on cooling, hence causing eventual loss of the smaller crystals [Hartel, 1998]) and, thus, resulting in the preservation of ice crystal size.

Most of the previous research completed in this area involves the study of ice recrystallization in quiescently frozen systems, that is, using a cold stage for microscope freezing and/or temperature cycling. However, little information is available about how the systems work in the industrial process (dynamically frozen by a scraped surface heat exchanger). The type of nucleation, introduction of air bubbles to the unfrozen phase and the kinetic energy transmitted to the ice cream mix during the freezing process are all variables that can affect the microstructure of the final product and, therefore, its functional properties. To better understand the functionality of hydrocolloids as stabilizers in ice cream, the microstructure and recrystallization rate of stabilizer solutions, frozen in a scraped surface heat exchanger and temperature cycled, were studied.

\section{MATERIALS AND METHODS}

\section{Preparation of Solutions}

The following aqueous solutions were prepared: $24 \%$ w/w sucrose (Fisher Scientific, Toronto, Canada) as control for sucrose solutions, $16 \% \mathrm{w} / \mathrm{w}$ sucrose $+14.7 \% \mathrm{w} /$ w milk solids nonfat ([MSNF] from skim milk powder from Gay Lea Foods, Guelph, ON, Canada) as control for sucrose/MSNF solutions, $24 \% \mathrm{w} / \mathrm{w}$ sucrose $+0.3 \%$ $\mathrm{w} / \mathrm{w}$ stabilizer and $16 \% \mathrm{w} / \mathrm{w}$ sucrose $+14.7 \% \mathrm{w} / \mathrm{w} \mathrm{MSNF}$ $+0.27 \% \mathrm{w} / \mathrm{w}$ stabilizer, using as stabilizers LBG, CMC, xanthan (Germantown Canada, Inc., Scarborough, $\mathrm{ON}$ ), and gelatin (250 Bloom; Cangel, Inc., Toronto, Canada), for a total of 10 solutions. Solute concentrations were chosen to reflect typical stabilizer:sugar ratios or stabilizer:MSNF:sugar ratios in an ice cream mix. Solutions were heated to $80^{\circ} \mathrm{C}$ for $30 \mathrm{~min}$, while 
stirring. Distilled water was added to bring the solutions to their original weight, and the solutions were cooled and stored at $4^{\circ} \mathrm{C}$ overnight. For statistical purposes, the different ice cream model solutions were prepared in triplicate.

\section{Freezing and Temperature Cycling Protocols}

Aliquots of $2 \mathrm{~L}$ were frozen in a scraped surface heat exchanger (Taylor Batch Freezer, model B733-32; Tekni-Craft, Rockton, IL). The mix was cooled down to a draw temperature of $-6^{\circ} \mathrm{C}$, and whipping was continued for a total of $15 \mathrm{~min}$. Overrun was measured and samples were drawn into cylindrical plastic containers $(250 \mathrm{ml})$ and immediately placed into a hardening room at $-35^{\circ} \mathrm{C}$ for storage. The containers were kept at this temperature overnight and then analyzed by microscopy.

For temperature cycling, three containers of each model solution were subsequently transferred to a cabinet freezer at $-6^{\circ} \mathrm{C}$. Each sample was subjected to a programmed heating and cooling cycle, during which the freezer was kept at $-6^{\circ} \mathrm{C}$ for $6 \mathrm{~h}$, then cooled at a rate of $2.33^{\circ} \mathrm{C} / \mathrm{h}$ to $-20^{\circ} \mathrm{C}$, held there for another $6 \mathrm{~h}$, and heated at a rate of $2.33^{\circ} \mathrm{C} / \mathrm{h}$ back to $-6^{\circ} \mathrm{C}$. This heating/cooling cycle was repeated five times on every sample, each cycle with a length of $24 \mathrm{~h}$.

\section{Brightfield Microscopy: Image Acquisition and Analysis}

Ice cream containers were transferred from the $-35^{\circ} \mathrm{C}$ walk-in freezer to a $-24^{\circ} \mathrm{C}$ storage room and then immediately prepared for microscopy analysis. All mechanical devices that were used for further treatment of the samples were precooled to $-24^{\circ} \mathrm{C}$. Cubes of approximately $1 \mathrm{~cm}^{3}$ were taken from the core section at the center of the container using a sharp knife and then fixed in the holder of a microtome (Spencer 820, American Optical, Markham, ON, Canada). From these cubes, a thin slice of $40 \mu \mathrm{m}$ was subsequently cut with the microtome. The sample was placed on a drop of isoamyl-butanol (previously cooled to $-24^{\circ} \mathrm{C}$ ) on a standard glass microscope slide, and a few more drops of isoamyl-butanol were added to the sample. Iso-amyl-butanol was used to disperse the ice crystals from the continuous phase of the ice cream, allowing for better image contrast. The microscope slide was then placed above liquid nitrogen in an insulated styrofoam container and transported immediately to an LTS 350 cold stage operated by a TP93 temperature programmer with liquid nitrogen pump (Linkam Scientific Instruments Ltd., Surrey, United Kingdom), which was previously programmed to a constant temperature of $-24^{\circ} \mathrm{C}$.
Images were acquired using a Sony XC-75 (Sony Corporation, Tokyo, Japan) video camera, mounted on an Olympus BH Polarized Light Microscope (Olympus, Tokyo, Japan) equipped with a green filter. A Scion LG-3 capture board was used for analogue-to-digital conversion and Scion image 1.62 for image acquisition (Scion Corporation, Fredrick, MD).

Brightfield images were acquired from the uncycled and cycled samples. For both, slides were prepared from three different containers. Several different fields were photographed to obtain at least 100 crystals for each slide and a total of 300 crystals for each sample. All images were calibrated by using images of a 1-mm stage micrometer that was acquired under conditions identical to the image being analyzed.

Measurements of ice crystal size were made with the image analysis software Object Image 1.62 (Vischer, Amsterdam, The Netherlands). By manually tracing the perimeter of the crystal with a computer mouse, the area of each crystal was automatically calculated by the software.

\section{Determination of Ice Crystal Growth}

Ice crystal size distributions were characterized by the logistic dose response model with a cumulative distribution of equivalent diameters, as previously described by Flores and Goff (1999b), obtaining the ice crystal diameter at $50 \%$ of the cumulative distribution function of the sample $\left(\mathrm{X}_{50}\right)$ and the slope of the cumulative distribution at $\mathrm{X}_{50}$. Statistical analysis of the data was carried out using Microsoft Excel 2000 (Microsoft Corporation, USA), ANOVA single-factor test. When significant effects were evidenced $(P<0.05)$ between sample treatments, $\mathrm{T}$ test (LSD) was used to compare the means of each parameter.

The rate of growth in the sample was calculated with the following equation:

$$
\begin{gathered}
\% \text { Rate of growth }=\left(\mathrm{X}_{50} \text { after cycling }-\right. \\
\left.\mathrm{X}_{50} \text { before cycling }\right) \times 100 / \mathrm{X}_{50} \text { before cycling }
\end{gathered}
$$

where the $\mathrm{X}_{50}$ values are taken from the averaged means of the triplicate runs.

\section{Microstructural Analysis}

Microstructural analysis was carried out by light microscopy (LM) and transmission electron microscopy (TEM). Freeze-substitution and low-temperature embedding techniques were used to preserve the microstructure of the sample.

Freeze-substitution and low-temperature embedding. Ice cream containers from cycled samples 
were transferred from a $-35^{\circ} \mathrm{C}$ walk-in freezer to a $-24^{\circ} \mathrm{C}$ storage room and then immediately prepared. Sample specimens (approximately $100 \mathrm{~mm}^{3}$ ) were cut from the inner bulk of the hardened ice cream with a surgical blade and immediately placed into liquid nitrogen $\left(-196^{\circ} \mathrm{C}\right)$. These sections were then broken with the surgical blade under slight force into $<1.0 \mathrm{~mm}^{3}$ pieces. The freeze-substitution technique of Robards and Sleytr (1985) was adapted for use. Frozen specimens of ice cream were transferred into vials containing a $3 \%(\mathrm{v} / \mathrm{v})$ glutaraldehyde, a fixative, in absolute methanol, which had been kept at $-196^{\circ} \mathrm{C}$ in liquid nitrogen. The vials were then placed in a $-90^{\circ} \mathrm{C}$ freezer for $14 \mathrm{~d}$, transferred to $-35^{\circ} \mathrm{C}$ for $2 \mathrm{~d}$, and later to $-24^{\circ} \mathrm{C}$ for 2 d. Specimens were washed twice in precooled $\left(-24^{\circ} \mathrm{C}\right)$ absolute ethanol for $30 \mathrm{~min}$, followed by the infiltration of the low-temperature embedding resin, LR Gold (Marivac Ltd., Nova Scotia, Canada), precooled to $-24^{\circ} \mathrm{C}$, as follows: one 30-min washing of a mix of $60 \%$ LR Gold monomer $+40 \%$ absolute ethanol, one 60 -min washing of a mix of $70 \%$ LR Gold monomer $+30 \%$ absolute ethanol, one 60 -min washing of $100 \%$ LR Gold monomer, one 60-min washing of $100 \%$ LR Gold monomer $+0.1 \%$ Benzil (initiator; Marivac Ltd., Nova Scotia, Canada) and lastly, a washing of $100 \%$ LR Gold monomer + $0.1 \%$ Benzil overnight. All the washings in the freezesubstitution protocol were made in a fume hood; vials were handled above styrofoam recipient containing liquid nitrogen or dry ice. In each step, after the change of solutions, the vials were transferred to a freezer cabinet at $-24^{\circ} \mathrm{C}$ for the completion of the substitution media. Specimens were then placed into gelatin capsules and covered with the mix 100\% LR Gold monomer + $0.1 \%$ Benzil. The gelatin capsules were tightly closed to avoid infiltration of oxygen. The resin-infiltrated samples were polymerized under $360 \mathrm{~nm}$ UV light at $-24^{\circ} \mathrm{C}$. Resin blocks were sectioned depending on the microscopy technique chosen: LM or TEM.

Microstructure by light microscopy. Resin blocks were sectioned at a thickness of $0.5 \mu \mathrm{m}$ using a LKB ultramicrotome (Leica Reichert Ultracut S., Vienna, Austria). To identify sections, a general stain, Toluidine Blue O (McCully, 1980), was applied. At least five sections were mounted on each glass slide for observation by LM. Differential staining for polysaccharides and proteins was accomplished by using the periodic acidleucobasic fuchsin (Schiff's reagent) and Amido Black 10B technique (Clark, 1981). Sections were observed on a light microscope (BX-60; Olympus, Tokyo, Japan). Brightfield images were acquired from cycled samples, from at least two blocks per treatment, three slides per block and 10 fields per slide.

Microstructure by transmission electron microscopy (TEM). Resin blocks obtained by freeze-substitu- tion and-low temperature embedding were also sectioned to a thickness of $90 \mathrm{~nm}$ using a LKB ultramicrotome. Sections were mounted on gold or copper grids (Marivac Ltd., Halifax, NS, Canada) depending on the poststaining procedure that was followed. Two different staining procedures were applied: silver proteinate (Thiery, 1967) and double staining with uranyl acetate and lead citrate (Lewis and Knight, 1977). Sections mounted in grids from either of the staining procedures were observed in a Hitachi H-7100 TEM (Tokyo, Japan) at $75 \mathrm{kV}$. At least two blocks of each sample were sectioned, and at least three sections per block and 10 fields per section per stain were viewed.

\section{RESULTS AND DISCUSSION}

\section{Ice Crystal Size Distributions and Rate of Growth (\%)}

Ice crystal size distributions obtained from image analysis were characterized by the values for the ice crystal equivalent diameter at $50 \%$ of the cumulative distribution $\left(\mathrm{X}_{50}\right)$ and the slopes at these $\mathrm{X}_{50}$ values. Statistical analysis by ANOVA showed significant differences between the averages of some of the treatments (Table 1).

The overall range of diameters after hardening confirms previous studies that suggested a mean ice crystal size of 45 to $50 \mu \mathrm{m}$ for ice cream after hardening (Donhowe et al., 1991). Values for $\mathrm{X}_{50}$ and the slopes at $\mathrm{X}_{50}$ of the solutions containing stabilizer either in the absence or presence of MSNF were not significantly different from the controls $(P>0.05)$. Thus, the presence of stabilizers in ice cream model solutions that have been frozen in dynamic conditions had no direct impact on the ice crystal size distribution after hardening, as has been reported by other research groups (MillerLivney and Hartel, 1997; Sutton and Wilcox, 1998a; Flores and Goff, 1999a). This may be attributed to the fact that stabilizers have no effect on the initial freezing properties of an ice cream mix.

The temperature cycling treatment produced an expected increase in the equivalent diameter $\left(\mathrm{X}_{50}\right)$ and a broadening of the distribution of the population characterized by a decrease of slope at the inflection point. Xanthan, LBG, and xanthan/MSNF in sucrose solutions resulted with the lowest values of ice crystal size $\left(\mathrm{X}_{50}\right)$ after temperature cycling. Significant differences in ice crystal size were found in these solutions when they were compared to their respective controls (Table 1). The rate of growth was calculated with reference to the extent to which the ice crystals increased in size after temperature cycling (Table 1). Values of slopes (data not shown) showed a similar trend as ice crystal sizes $\left(\mathrm{X}_{50}\right)$, with xanthan having a significantly higher 
Table 1. $\mathrm{X}_{50}{ }^{1}$ values (after hardening and after temperature cycling ${ }^{2}$ ) and ice crystal growth ${ }^{3}$ of stabilizer $(0.3 \% \mathrm{w} / \mathrm{w})$ solutions containing sucrose $(24 \% \mathrm{w} / \mathrm{w})$ and sucrose $(16 \% \mathrm{w} / \mathrm{w})$ plus MSNF $(14.7 \% \mathrm{w} / \mathrm{w})$ frozen by scraped surface heat exchanger.

\begin{tabular}{|c|c|c|c|c|c|c|}
\hline \multirow[b]{3}{*}{ Stabilizer } & \multicolumn{3}{|c|}{ Sucrose solutions, $\mathrm{X}_{50}{ }^{1}(\mathrm{~mm})$} & \multicolumn{3}{|c|}{ Sucrose/MSNF solutions, $\mathrm{X}_{50}(\mathrm{~mm})$} \\
\hline & \multicolumn{2}{|c|}{$\mathrm{X}_{50}^{1}(\mathrm{~mm})$} & \multirow[b]{2}{*}{$\begin{array}{l}\text { Ice crystal } \\
\text { growth }(\%)^{3}\end{array}$} & \multicolumn{2}{|c|}{$\mathrm{X}_{50}(\mathrm{~mm})$} & \multirow[b]{2}{*}{$\begin{array}{l}\text { Ice crystal } \\
\text { growth }(\%)\end{array}$} \\
\hline & $\begin{array}{l}\text { Afte } \\
\text { hardening }\end{array}$ & $\begin{array}{l}\text { After } \\
\text { cycling }^{2}\end{array}$ & & $\begin{array}{l}\text { After } \\
\text { hardening }\end{array}$ & $\begin{array}{l}\text { After } \\
\text { cycling }\end{array}$ & \\
\hline None & $47.9^{\mathrm{a}, \mathrm{C}}$ & $104.1^{\mathrm{a}, \mathrm{A}}$ & 117.2 & $41.4^{\mathrm{a}, \mathrm{D}}$ & $89.2^{\mathrm{a}, \mathrm{B}}$ & 115.4 \\
\hline CMC & $45.7^{\mathrm{a}, \mathrm{B}}$ & $96.8^{\mathrm{a}, \mathrm{b}, \mathrm{A}}$ & 111.6 & $43.3^{\mathrm{a}, \mathrm{B}}$ & $88.5^{\mathrm{a}, \mathrm{A}}$ & 104.3 \\
\hline LBG & $48.7^{\mathrm{a}, \mathrm{B}}$ & $90.7^{\mathrm{b}, \mathrm{A}}$ & 86.1 & $42.0^{\mathrm{a}, \mathrm{C}}$ & $84.4^{\mathrm{a}, \mathrm{A}}$ & 101.3 \\
\hline Gelatin & $45.2^{\mathrm{a}, \mathrm{C}}$ & $98.5^{\mathrm{a}, \mathrm{b}, \mathrm{A}}$ & 117.7 & $41.8^{\mathrm{a}, \mathrm{C}}$ & $85.9^{\mathrm{a}, \mathrm{B}}$ & 105.3 \\
\hline Xanthan & $44.4^{\mathrm{a}, \mathrm{C}}$ & $72.9^{\mathrm{c}, \mathrm{A}}$ & 64.1 & $38.9^{\mathrm{a}, \mathrm{D}}$ & $63.8^{\mathrm{b} . \mathrm{B}}$ & 63.9 \\
\hline
\end{tabular}

${ }^{\mathrm{a}, \mathrm{b}, \mathrm{c}}$ Values for each parameter with the same letter in the same column do not differ $(P>0.05)$.

A,B,C,D Values for each parameter with the same letter in the same row do not differ $(P>0.05)$.

${ }^{1}$ Ice crystal diameter at $50 \%$ of the cumulative distribution.

${ }^{2}$ Five cycles from $-20^{\circ} \mathrm{C}$ to $-6^{\circ} \mathrm{C}$.

${ }^{3}$ Expressed as \% of uncycled samples.

slope and, therefore, a noticeably smaller span of ice crystal distribution than all other solutions. This phenomenon is related to the ability of the xanthan stabilizer to promote the melt-regrow instead of the meltdiffuse-grow mechanism of recrystallization under temperature cycling conditions. The reduction of ice recrystallization rates by the addition of stabilizers in model solutions and in ice cream mixes, frozen by dynamic conditions, has been reported as well by several researchers: Miller-Livney and Hartel, 1997; Sutton and Wilcox, 1998a; Flores and Goff, 1999b. However, the vastly different formulations evaluated, freezing and temperature cycling protocols, and methods for quantifying the ice crystal size applied in those studies makes direct comparisons of results difficult.

All solutions containing MSNF resulted in lower values of ice crystal size $\left(\mathrm{X}_{50}\right)$ than their corresponding sucrose solutions (Table 1). Proteins exerted a cryoprotective effect in the samples that resulted in less growth of ice crystals, as previously reported by Goff et al. (1999).

\section{Microstructural Analysis}

Freeze-substitution and low-temperature embedding techniques have proven to be effective sample preparation techniques, as the original structure of the specimen is preserved. However, potential artefacts (such as sample shrinking, loss of soluble substances, and heterogeneous resin embedding) can cause misinterpretation. Simultaneous application of light microscopy and transmission electron microscopy techniques validate results between each other, helping in the understanding of stabilizer functionality.

Microstructure by light microscopy. The Toluidine Blue $\mathrm{O}$ staining of sections obtained from the blocks prepared by freeze-substitution and low-temperature embedding allowed the identification of the sample within the resin and contributed preliminary information about the microstructural features present in the samples. The leucobasic fuchsin protocol was applied to selectively stain (in red) the polysaccharides present in the sample. Amido-black is a protein stain that gives a black color. The efficiency of these methods was proven by observing light microscopy slides with sections prepared from sucrose and sucrose/MSNF solutions containing either 0 or $0.3 \%$ LBG. The acquisition of color micrographs allowed the identification of very specific structures stained brilliant red after the leucobasic fuchsin staining (features that were not present in the controls). The rest of the sample showed a pale pink color. After the application of amido-black to the samples containing MSNF, post-leucobasic-fuchsin staining clearly distinguished white zones where the crystals existed. The serum phase showed a very distinctive phase separation where red agglomerates of polysaccharides were dispersed within the black serum phase composed mainly by proteins (caseins and whey proteins). For microstructural comparisons, all the samples were subjected to the stated double staining, observed by light microscopy and photographed using black and white film. Although the physical color of the staining procedure could not be identified due to equipment limitations, this allowed for a considerable increase in resolution.

Representative light micrographs obtained after double staining (leucobasic fuchsin and amido-black) of sections for all treatments are shown in Figure 1. A gel-like structure was observed in sucrose solutions containing LBG. The ice crystal borders could not be identified, suggesting that the network structure did not completely cover all the unfrozen phase of the sample. This 


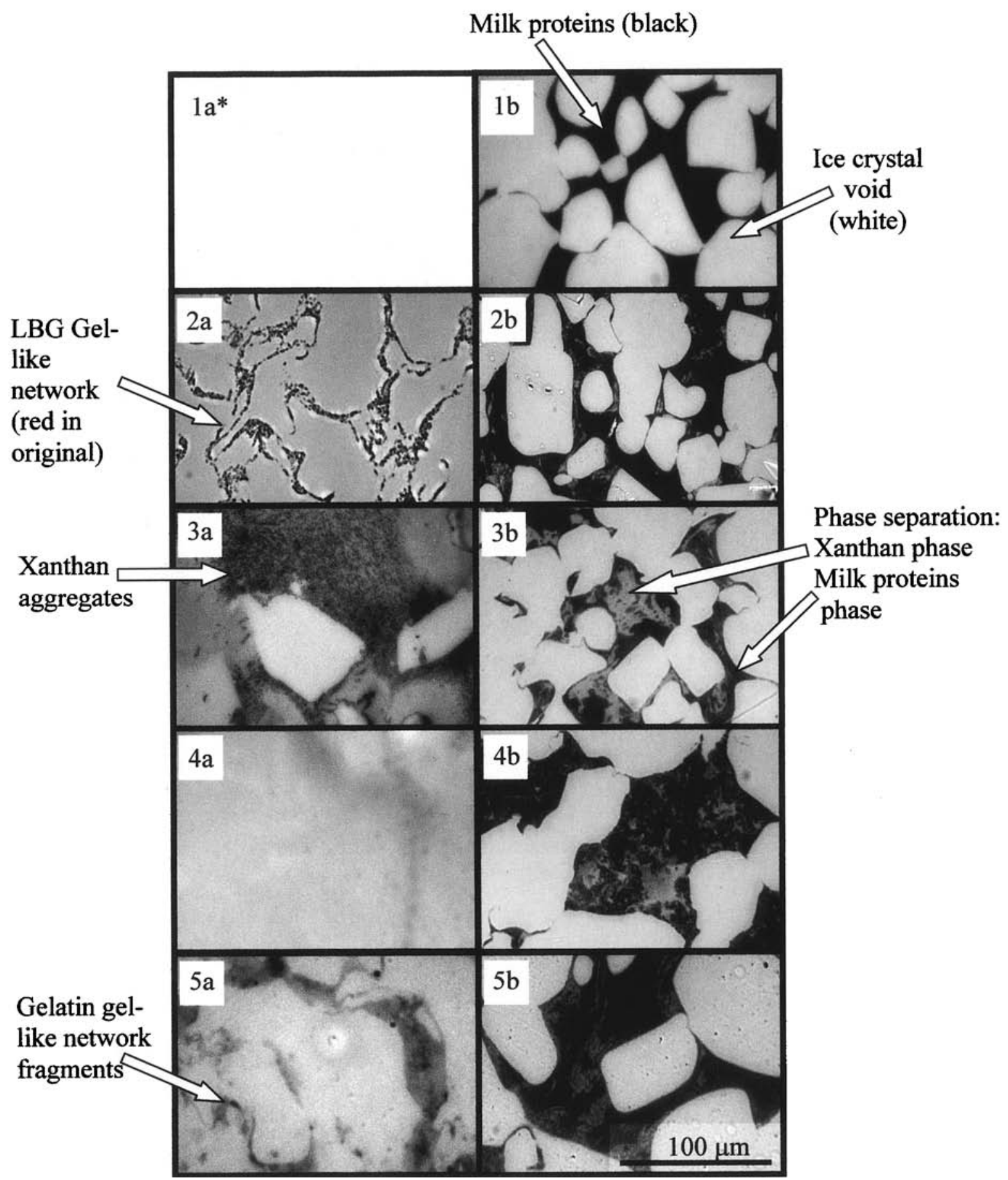

Figure 1. Representative images from a) sucrose and b) sucrose with MSNF solutions containing either no or $0.3 \%$ stabilizer, frozen by dynamic conditions, subjected to temperature cycling and observed by light microscopy after double staining (leucobasic fuchsin and amidoblack) procedure: 1) no stabilizer, 2) LBG, 3) xanthan, 4) CMC, and 5) gelatin. *Image acquisition was not possible because the preparation technique was not suitable for this sample.

observation rejects the theory of stabilizer adsorption to the ice crystal interface as a mechanism of stabilizer action proposed by other researchers (Sutton et al., 1996a,b and 1997). Some fragments of a gelatin gel, possibly broken by the dynamic movement of the scraped surface heat exchanger, were observed. No ice crystal borders were observed in this system either. In the case of xanthan in sucrose solution, the stabilizer 
was distributed in the unfrozen serum phase, delimiting the ice crystal interface, and thus allowing the identification of the ice crystals in the image. Some xanthan aggregates were detected in localized regions of the unfrozen phase. No structural features were detected in the sample containing CMC.

The representative images of stabilizer-sucrose solutions containing MSNF showed phase separation between biopolymers in the unfrozen serum phase. Gelatin and milk proteins were differentiated because of their different susceptibilities to the stain (amidoblack). In all of these images, milk proteins were localized, surrounding the interface of the ice crystals, which suggests that their role at retarding recrystallization may be to act as water-holding molecules at the interface while polysaccharides reinforce the microviscosity of the unfrozen phase.

Microstructure by transmission electron microscopy. The staining procedure with silver proteinate was not effective. Further research is needed to evaluate different variables, such as time of residence of the sections in the stain, temperatures, concentrations, or other factors that could affect the results. The most successful staining technique was the uranyl acetate followed by lead citrate. Figures 2 and 3 show representative micrographs from each one of the systems studied, at two different magnifications.

Verifying the results obtained by the light microscopy technique, the systems in which a gel-like structure was detected were gelatin and LBG in sucrose solutions (Figure 2). Xanthan showed small aggregates with a very fine structure and an approximate diameter of $0.2 \mu \mathrm{m}$. The images acquired from the gelatin solution showed big fragments of thick strands that were not always connected. No structural features at any magnification could be observed in the sample containing CMC.

In Figure 3, images acquired from the sucrose solutions containing MSNF show $\mathrm{CN}$ micelles and some gel-like structures formed by the stabilizers. In these images, phase separation originating from thermodynamic incompatibility between protein and polysaccharide is evident at a low magnification $(10 \mathrm{~K} \times)$. An order in the degree of phase separation could be qualitatively established from these images: none for the control, low for LBG and CMC, and high for xanthan.

Interestingly, more information can be obtained from images at higher magnifications ( 60 to $80 \mathrm{~K} \times$ ). The gellike structure of LBG, within the regions depleted of $\mathrm{CN}$ micelles, was detected. Some aggregation in the CMC sample was also observed. Xanthan aggregates, similar to the ones found in the xanthan/sucrose solution, were not detected in the presence of proteins. Some smaller aggregates were observed, however. The im- ages acquired from samples containing gelatin and milk proteins suggest that the unfrozen phase in these systems is constituted by a composite gel of gelatin with CN micelles trapped within.

In summary, the only stabilizers that significantly retarded ice crystal growth in samples frozen by dynamic conditions after temperature cycling were LBG without MSNF and xanthan with and without MSNF. The presence of gel-like networks was observed in LBG and gelatin, both with and without MSNF. Therefore, gelation of the polymer is not the only mechanism of stabilizer action. Instead, the present study suggests that the ability of stabilizers in retarding recrystallization could be a result of either or both factors, a microviscosity increase in the surroundings of the ice crystals (i.e., xanthan) and/or the steric hindrance offered by a stabilizer gel-like network (i.e., LBG). In the presence of milk proteins, thermodynamic incompatibility between biopolymers promotes localized high concentrations of proteins, located at the ice crystal interface, exerting a water-holding action that significantly enhances the stabilizer effect in retarding ice recrystallization. Qualitatively, solution heterogeneity (phase separation) seems to be directly proportional to ice crystal growth inhibition (i.e., xanthan + MSNF maximum).

Considering previous research (Regand and Goff, accepted) and the results obtained from this study, the efficiency of a stabilizer in controlling ice crystal growth in sucrose solutions with or without MSNF frozen under different conditions (quiescent vs. dynamic) and temperature cycled have been compared in Table 2 . The effects of freezing method and/or presence of proteins in the recrystallization inhibition behavior of LBG were evidenced by these two studies. According to Regand and Goff (under review) in quiescent conditions, a homogeneous LBG gel-like structure with small pores was identified by fluorescence microscopy. This structure was located surrounding the ice crystals and did not shrink with cycling, which could be indicative of a rigid structure. After extensive cycling (high frequency cycles at high temperature), the self-association of the hydrocolloid could promote some syneresis and the waterholding capacity of the stabilizer could be decreased. With the addition of milk proteins (LBG/MSNF solutions), the protein molecules could surround the ice crystals even when they shrank during the melting steps, providing additional water-holding that resulted in reduction of ice recrystallization.

In dynamic conditions (present research), the LBG network observed by LM and TEM in LBG/sucrose solutions after cycling showed a completely different structure than in quiescent conditions (Regand and Goff, accepted). In this case, the pores of the gel-like network were bigger and the structure was not so homogeneous. 


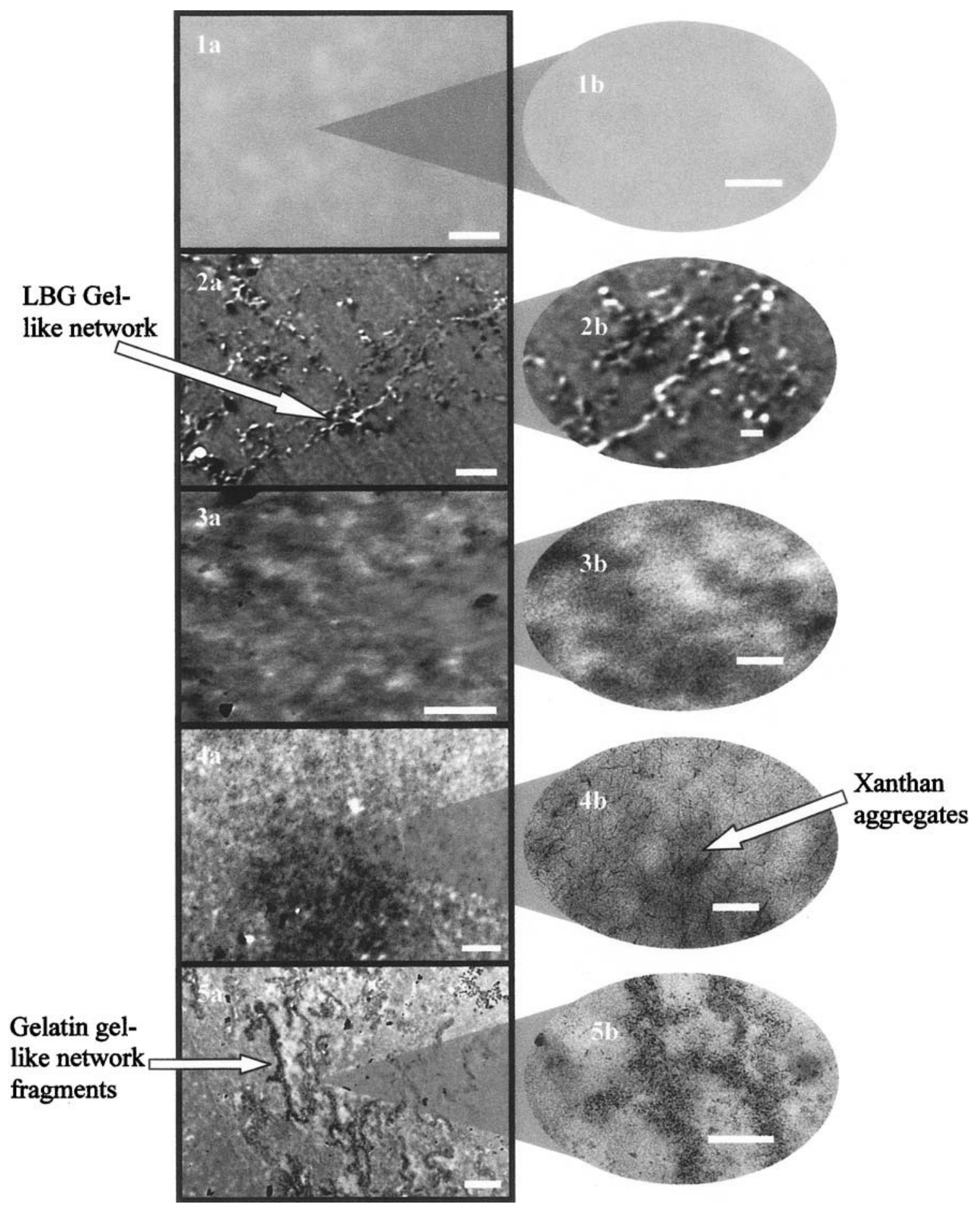

Figure 2. Representative micrographs of uranyl acetate-lead citrate stained sections obtained after thin-sectioning blocks of freezesubstituted and low-temperature embedded sucrose solutions containing either no or $0.3 \%$ stabilizer, frozen in a surface scraped heat exchanger and temperature cycled, observed by TEM at two magnifications: a) bar $=1 \mu \mathrm{m}, \mathrm{b})$ bar $=0.2 \mu \mathrm{m}, 1)$ no stabilizer, 2) LBG, 3) CMC, 4) xanthan, and 5) gelatin.

Probably the inclusion of air, the continuous agitation during freezing and the less aggressive cycling protocol (lower frequency cycles at lower temperature than in quiescent conditions) reduced the self-association of the biopolymer, allowing it to hold some water that resulted in a small reduction of ice crystal growth. With the 


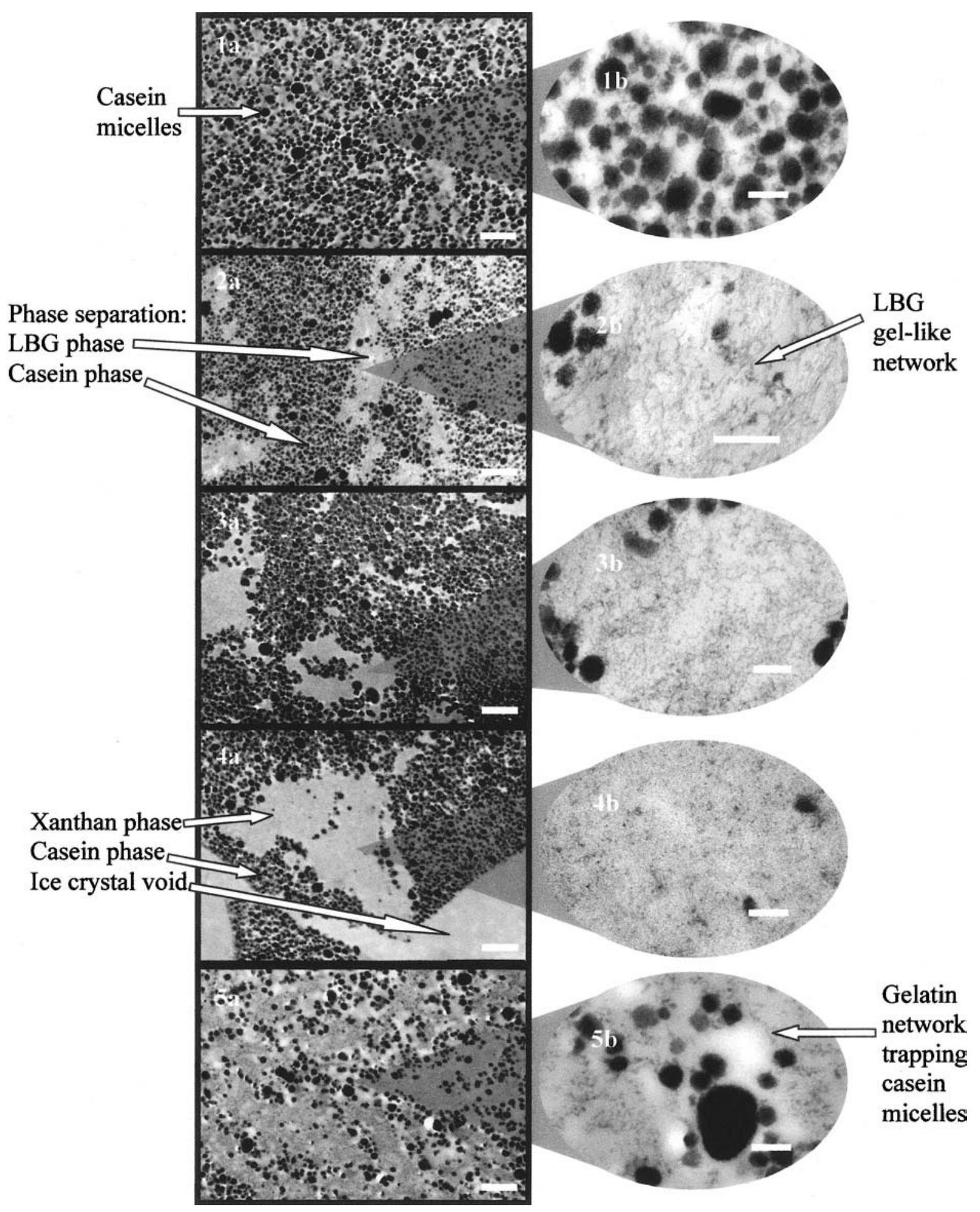

Figure 3. Representative micrographs of uranyl acetate-lead citrate stained sections obtained after thin-sectioning blocks of freezesubstituted and low-temperature embedded sucrose/MSNF solutions containing either no or $0.3 \%$ stabilizer, frozen in a surface scraped heat exchanger and temperature cycled, observed by TEM at two magnifications: a) 10 to 20K, b) 60 to 100K, 1) no stabilizer, 2) LBG, 3) CMC, 4) xanthan, and 5) gelatin. 
Table 2. Comparison of stabilizer ability in retarding ice recrystallization in solutions with or without MSNF when the samples have been frozen quiescently (in a cold stage) [reported in Regand and Goff, accepted] or dynamically (in a scraped surface heat exchanger).

\begin{tabular}{llllll}
\hline & \multicolumn{2}{c}{ Sucrose solutions } & & \multicolumn{2}{c}{ Sucrose/MSNF solutions } \\
\cline { 2 - 3 } \cline { 5 - 6 } & Quiescent & Dynamic & & Quiescent & Dynamic \\
\hline CMC & No $^{1}$ & No & & Yes & No \\
LBG & No & Yes & & Yes & No \\
Gelatin & No & No & & No & No \\
Xanthan & Yes & Yes & & Yes & Yes \\
Alginate & Yes & NS & Yes & NS \\
Carrageenan & No & NS & Yes & NS \\
\hline
\end{tabular}

${ }^{1}$ No significant effect in retarding recrystallization $(P>0.05)$.

${ }^{2}$ Significant effect in retarding recrystallization $(P<0.05)$.

${ }^{3}$ Not studied.

addition of proteins, the water-holding effect is again considerably enhanced, reported by a lower ice crystal size in LBG/MSNF solutions than in LBG solutions. However, the water-holding capabilities of the milk proteins per se, showed in the control, were so high that the additional steric/water-holding effect offered by the LBG was not significantly evident.

Regarding the use of CMC as stabilizer, a positive effect from the addition of milk proteins in the ability of the hydrocolloid to retard recrystallization in sucrose solutions was evident for both freezing methods. However, CMC/MSNF was only significantly effective in solutions frozen under quiescent conditions. In dynamic conditions, the ice crystal growth (\%) in the CMC/MSNF sample was lower than in the control, but the difference was too small to be significant.

For the rest of the stabilizers, the same trend in stabilizer effectiveness was observed in both freezing methods.

\section{CONCLUSIONS}

The application of LM and TEM techniques to the analysis of the microstructure of stabilizer (CMC, xanthan, LBG, and gelatin) in sucrose and sucrose/MSNF solutions frozen in a scraped surface heat exchanger and temperature cycled was successfully achieved. Differential staining for polysaccharides and proteins allowed the identification of stabilizer gel-like networks in LBG, gelatin, and gelatin/MSNF sucrose solutions. Proteins rather than polysaccharides were located at the ice crystal solution interface. Ice crystal growth was significantly retarded in solutions containing LBG, xanthan and xanthan/MSNF. Therefore, as with freezing under quiescent conditions (Regand and Goff, accepted), gelation of the polymer is not the only mechanism of stabilizer to retard ice crystal growth. It is suggested that water-holding by stabilizer and proteins, and in some cases steric hindrance induced by a stabilizer gel-like network, caused a reduction in the kinetics of the ice recrystallization phenomena and promoted mechanisms of melt-re-grow instead of melt-diffuse-grow, thus resulting in the preservation of the ice crystal size and in a small span of the ice crystal size distribution. Differences in stabilizer effectiveness are possibly related with the flexibility of the gel-like network, when it is present, or the ability of the solution to collapse together with the ice crystal during ice crystal melting. The most efficient stabilizer would be the one giving sufficient microviscosity to the solution and steric hindrance to retard water diffusion to other crystals, but at the same time, it would need to have optimum visco-elastic properties that are not affected by heat shock. An important participation of milk proteins in controlling ice crystal growth at the surroundings of the ice crystal interface was evidenced by the microscopy techniques utilized in the study.

\section{ACKNOWLEDGMENTS}

The authors wish to thank Sandy Smith and Ken Baker for their technical support and intellectual contributions to the present research. Appreciation is expressed as well to Christine Epp, a visiting student from TUM, Weihenstephan, Germany, for her valuable contribution to this study. Thanks as well to CONACYT Mexico and the Natural Sciences and Engineering Research Council of Canada for the financial support that made possible this investigation.

\section{REFERENCES}

Blond, G. 1988. Velocity of linear crystallization of ice in macromolecular systems. Cryobiology. 25:61-66.

Bolliger, S., H. Wildmoser, H. D. Goff, and B. W. Tharp. 2000. Relationships between ice cream mix viscoelasticity and ice crystal growth in ice cream. Int. Dairy J. 10:791-797.

Budiaman, E. R., and O. R. Fennema. 1987. Linear rate of water crystallization as influenced by viscosity of hydrocolloid suspensions. J. Dairy Sci. 70:547-554. 
Caldwell, K. B., H. D. Goff, and D. W. Stanley. 1992a. A low temperature scanning electron microscopy study of ice cream. I. Techniques and general microstructure. Food Structure. 11:1-9.

Caldwell, K. B., H. D. Goff, and D. W. Stanley. 1992b. A low temperature scanning electron microscopy study of ice cream. II. Influence of selected ingredients and processes. Food Structure. 11:11-23.

Clark, G. 1981. Staining Procedures. 4th ed. Williams \& Wilkins. Baltimore, MD.

Donhowe, D. P., and R. W. Hartel. 1996a. Recrystallization of ice during bulk storage of ice cream. Int. Dairy J. 6:1209-1221.

Donhowe, D. P., and R. W. Hartel. 1996b. Recrystallization of ice in ice cream during controlled accelerated storage. Int. Dairy J. 6:1191-1208.

Donhowe, D. P., R. W. Hartel, and R. C. Bradley. 1991. Ice crystallization processes during manufacture and storage of ice cream. J. Dairy Sci. 74:3334-3344.

Flores, A. A., and H. D. Goff. 1999a. Ice crystal size distributions in dynamically frozen model solutions and ice cream as affected by stabilizers. J. Dairy Sci. 82:1399-1407.

Flores, A. A., and H. D. Goff. 1999b. Recrystallization in ice cream after constant and cycling temperature storage conditions as affected by stabilizers. J. Dairy Sci. 82:1408-1415.

Goff, H. D., K. B. Caldwell, D. W. Stanley, and T. J. Maurice. 1993. Influence of polysaccharides on the glass transition in frozen sucrose solutions and ice cream. J. Dairy Sci. 76:1268-1277.

Goff, H. D., D. Ferdinando, and C. Schorsch. 1999. Fluorescence microscopy to study galactomannan structure in frozen sucrose and milk protein solutions. Food Hydrocoll. 13:353-362.

Hagiwara, Y., and R. W. Hartel. 1996. Effect of sweetener, stabilizer and storage temperature on ice recrystallization in ice cream. J. Dairy Sci. 79:735-744.

Hartel, R. W. 1998. Mechanisms and kinetics of recrystallization in ice cream. Pages 287-319 in The Properties of Water in Foods. D. S. Reid, ed. Blackie Academic and Professional, London, United Kingdom.
Lewis, P. R., and D. P. Knight. 1977. Staining Methods for Sectioning Material. A. M. Glauert, ed. Elsevier/North-Holland Biomedical Press, Cambridge, United Kingdom.

Martin, D. R., S. Ablett, A. Darke, R. L. Sutton, and M. E. Sahagian. 1999. Diffusion of aqueous sugar solutions as affected by locust bean gum studied by NMR. J. Food Sci. 64:46-49.

McCully, M. E. 1980. Plant Histochemistry. Unpublished report. McMaster University, Hamilton, ON, Canada.

Miller-Livney, T., and W. Hartel. 1997. Ice recrystallization in ice cream: Interactions between sweeteners and stabilizers. J. Dairy Sci. 80:447-456.

Muhr, A. H., and J. M. Blanshard. 1986. Effect of polysaccharide stabilizers on the rate of growth of ice. J. Food Technol. 21:683-710.

Regand, A., and H. D. Goff. Structure and ice recrystallization in frozen stabilized ice cream model systems. Food Hydrocoll. (Accepted).

Robards, A. W., and U. B. Sleytr. 1985. Low temperature methods in biological electron microscopy. Pages 461-500 in Practical Methods in Electron Microscopy. Vol. 10. A. M. Glauert, ed. Elsevier, Cambridge, United Kingdom.

Sutton, R. L., and J. Wilcox. 1998a. Recrystallization in ice cream as affected by stabilizers. J. Food Sci. 63:104-107.

Sutton, R. L., and J. Wilcox. 1998b. Recrystallization in model ice cream solutions as affected by stabilizer concentration. J. Food Sci. 63:9-11.

Sutton, R. L., A. Lips, and G. Piccirillo. 1996a. Recrystallization in aqueous fructose solutions as affected by locust bean gum. J. Food Sci. 61:746-748.

Sutton, R. L., A. Lips, G. Piccirillo, and A. Sztehlo. 1996b. Kinetics of ice recrystallization in aqueous fructose solutions. J. Food Sci. 61:741-745

Sutton, R. L., D. Cooke, and A. Russell. 1997. Recrystallization in sugar/stabilizer solutions as affected by molecular structure. J. Food Sci. 62:1145-1149.

Thiery, J. P. 1967. Demonstration of polysaccharides on thin sections in electron microscopy. J. Microscopie 6:987-1018. 